\title{
Carotid Intima-Media Thickness Studies: Study Design and Data Analysis
}

\author{
Sanne A.E. Peters, Michiel L. Bots \\ Julius Center for Health Sciences and Primary Care, University Medical Center Utrecht, Utrecht, The Netherlands
}

Background Carotid intima-media thickness (CIMT) measurements have been widely used as primary endpoint in studies into the effects of new interventions as alternative for cardiovascular morbidity and mortality. There are no accepted standards on the use of CIMT measurements in intervention studies and choices in the design and analysis of a CIMT study are generally based on experience and expert opinion. In the present review, we provide an overview of the current evidence on several aspects in the design and analysis of a CIMT study on the early effects of new interventions.

Summary of Issues A balanced evaluation of the carotid segments, carotid walls, and image view to be used as CIMT study endpoint; the reading method (manual or semi-automated and continuously or in batch) to be employed, the required sample size, and the frequency of ultrasound examinations is provided. We also discuss the preferred methods to analyse longitudinal CIMT data and address the possible impact of, and methods to deal with missing and biologically implausible CIMT values.

Conclusions Linear mixed effects models are the preferred way to analyse CIMT data and do appropriately handle missing and biologically implausible CIMT values. Furthermore, we recommend to use extensive CIMT designs that measure CIMT at regular points during the multiple carotid sites as such approach is likely to increase the success rates of CIMT intervention studies designed to evaluate the effects of new interventions on atherosclerotic burden.

Keywords Carotid intima-media thickness; Trials; Study design; Data analysis; Atherosclerosis

\author{
Correspondence: Sanne A.E. Peters \\ Julius Center for Health Sciences and \\ Primary Care, Stratenum 6.131, \\ University Medical Center Utrecht, \\ Heidelberglaan 100, 3584 CX, Utrecht, \\ The Netherlands \\ Phone: $+31-887559380$ \\ Fax: +31 887555485 \\ E-mail: s.a.e.peters@umcutrecht.nl
}

Received: September 19, 2012

Revised: January 9, 2013

Accepted: January 9, 2013

The authors have no financial conflicts of interest.

\section{Introduction}

Atherosclerosis is a slow and progressive disease of the arterial wall that underlies the majority of cardiovascular events. ${ }^{1}$ Even though atherosclerosis may remain clinically silent for decades, it can be non-invasively assessed from early to late stages of the disease process using different imaging techniques (Figure 1). B-mode ultrasound is one of those imaging techniques which is frequently used to assess atherosclerosis in a safe, inexpensive, reliable, and reproducible manner. B-mode ultrasound measurements of the carotid intima-media thickness (CIMT) have been first described in 1986 by Pignoli et al. in an in vitro study of common carotid arteries. ${ }^{2}$ The investigators showed that the distance between the lumen and intima interface of the common carotid artery from pathologic examination did not differ from distance between the echogenic lines seen on the Bmode ultrasound measurement from the same sample, suggesting that B-mode ultrasound could be used to measure CIMT in vivo. At present, CIMT is an accepted measure of atherosclerosis that has frequently been used in observational studies to study the causes and consequences of atherosclerosis. ${ }^{3-5}$ In addition, numerous randomized controlled trials have used rate of change 


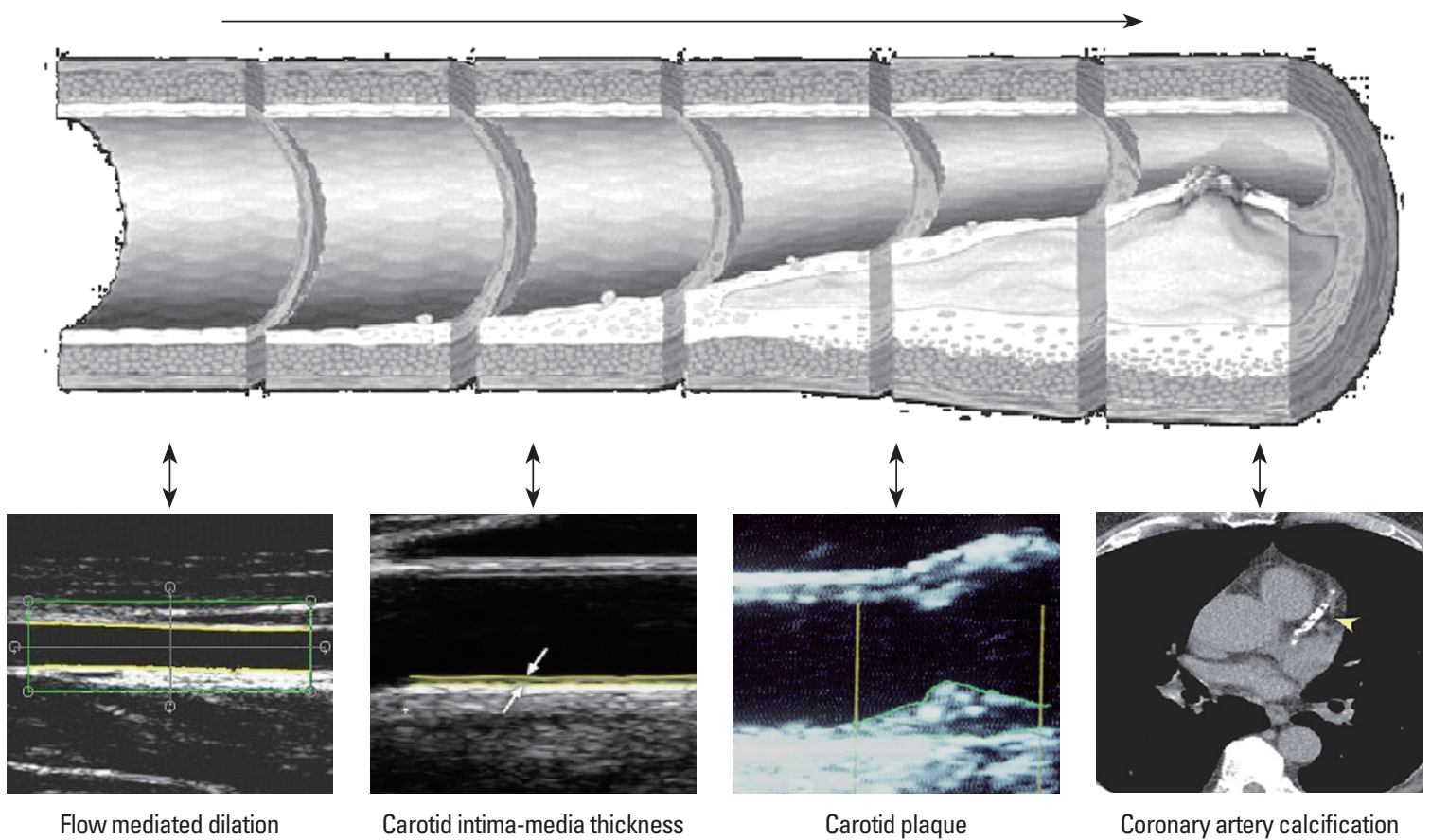

Figure 1. Imaging of atherosclerosis in sequential stages of the disease process.

in CIMT as alternative endpoint for cardiovascular disease events to evaluate the effects of new interventions. ${ }^{6-13}$ The main advantage of using CIMT as an outcome variable in studies is the considerable increase in efficacy in sample size and duration of follow-up when compared to studies using morbidity and mortality as primary outcome. Nevertheless, while CIMT measurements are increasingly being used, there are still no accepted standards on the use of CIMT measurements in various research areas. Hence, choices in the design and analysis of a CIMT study are generally based on experience and expert opinion rather than on solid evidence. Some methodological issues have begun to be addressed and the results from these studies do provide evidence for the most optimal approach to design and statistically analyse a CIMT study into the early effects of a new intervention on atherosclerosis before the start of a large morbidity and mortality study. ${ }^{14-16}$ In the present review, we provide an overview of the current evidence on the design and analysis of a CIMT study on the early effects of new interventions.

\section{Study endpoint}

CIMT is a common term for many different types of arterial measurements and there is a lot of heterogeneity across studies regarding the measurements that are included in the study endpoint. Figure 2 provides a schematic representation of the CIMT measurements that could be included in the most extensive

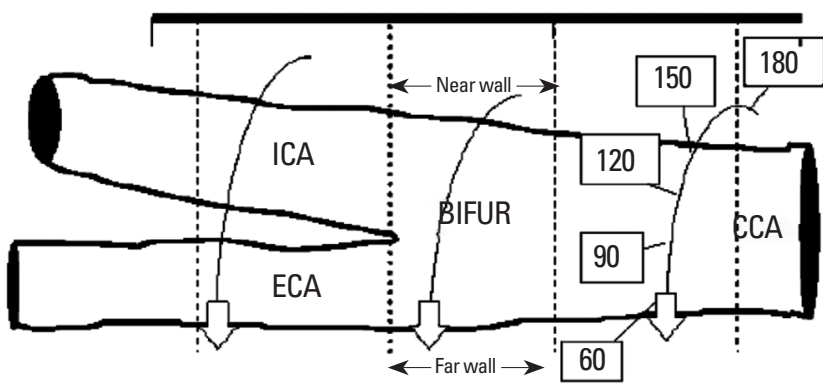

Figure 2. Graphical representation of circumferential assessment of the artery sites. The values from 60 to 180 represent the standardized angles of interrogation. BIFUR, carotid bifurcation; CCA, common carotid artery; ECA, external carotid artery; ICA, internal carotid artery.

protocols. Differences between protocols involve (1) the arterial segments (e.g. the common carotid artery, the carotid bifurcation, and/or the internal carotid artery); (2) the carotid walls (far wall or both the near and far wall); and (3) the angle view (or view) to be used (one optimal angle, the thickest angle, or multiple angles).

\section{Carotid segments}

Some studies restricted their measurements to the common carotid artery alone, whereas ultrasound protocols including measurements from the carotid bifurcation and the internal carotid artery are used in the more extensive studies. Main arguments to restrict ultrasound protocols to measurements of the 
common carotid artery only are that common CIMT data collection is very reproducible and nearly always complete whereas the carotid bifurcation and the internal carotid artery are thought to be a source of missing data and less reproducible. Indeed, reproducibility of the CIMT measurements in earlier cohort studies was $<0.75$ for the carotid bifurcation and the internal carotid artery whereas it was 0.85 or higher for the common carotid artery. ${ }^{17}$ In addition, completeness rates for the carotid bifurcation and internal carotid artery were $83 \%$ and $56 \%$ of the individuals in the Rotterdam Study, respectively, whereas measurements of the common carotid artery could be obtained in 97\% of the individuals. ${ }^{18}$ However, CIMT measurements have advanced importantly and recent studies indicate that data completeness and reproducibility are also high for the carotid bifurcation and the internal carotid artery. ${ }^{15,19,20}$ Therefore, expected difficulties in visualizing the carotid bifurcation and the internal carotid artery should not be the main argument to restrict the ultrasound protocol to the common carotid artery alone. Instead, as the aim of the use of CIMT in intervention studies is to determine the effect of an intervention on atherosclerosis and cardiovascular risk, one should choose the (combination of) arterial segment(s) that best reflects atherosclerosis and/or cardiovascular risk. Indeed, there are examples of therapies that proved to be effective in morbidity and mortality studies that failed to show an intervention effect on the CIMT of the common carotid artery whereas a beneficial effect was found on the CIMT endpoint that included the carotid bifurcation and the internal carotid artery. ${ }^{15}$ Recent studies that were set out to determine the best ultrasound protocol in terms of carotid segments to be included and showed mixed results. ${ }^{14,21,22}$ Measuring the near and far wall of the common carotid artery at multiple angles alone was superior to also measuring the carotid bifurcation and internal carotid artery in healthy individuals and in familial hypercholesterolemia patients, whereas the three segment approach was superior to the common carotid artery approach alone in individuals with mixed dyslipidemia and asymptomatic subclinical atherosclerosis. Thus, although measurement of the common carotid artery alone may be sufficient in some situations, intervention effects may be missed and important new therapies may not be further developed and evaluated in other situations. Importantly, as data collection on all arterial segments also allows for evaluation of the common carotid artery alone, but not the other way around, extensive protocols may prevail.

\section{Carotid walls}

It is generally recognized that the far wall CIMT accurately reflects the true thickness of the carotid wall whereas the near wall is only an approximation of the true thickness. ${ }^{2,23,24}$ There is no published evidence that shows that the combined near and far wall common CIMT is superior to the far wall common CIMT alone in the relation with prevalent or incident disease. ${ }^{25}$ Yet, near wall CIMT measurements are nearly always complete and are as feasible and as reproducible as far wall CIMT measurements. ${ }^{19,20}$ Also, the combination of near and far wall CIMT measurements has shown to be superior to far wall CIMT measurements alone in a number of intervention studies. ${ }^{14,21,22}$ This superiority could be explained by the reduction in random measurement error and the subsequent improvement in precision after combining of the near and far wall measurements. Hence, measurement of near wall CIMT may yield valuable information and does increase statistical power and should not be discarded.

\section{Image view}

CIMT ultrasound protocols also differ in the view (e.g. the angle) that is used to measure CIMT. Some studies measure CIMT only once at each measurement site and choose between an image in which the intima and media interfaces are most clear (i.e. single optimal B-mode image), ${ }^{26}$ or search for the point with the thickest CIMT (e.g. the highest burden of atherosclerosis). ${ }^{4}$ Others choose for multiple optimal B-mode images, ${ }^{27}$ or measured CIMT from multiple images that were taken from various standardized angles of interrogation which allows for measurement at exactly the same location over time. ${ }^{9}$ When one is interested in the rate of change in CIMT and the effects of an intervention, a few studies have been published that assessed which approach is most favourable. These studies showed that extensive ultrasound protocols including near and far wall measurements from 2 or more angles provide a better balance between high reproducibility, large progression rates, and large and precise intervention effects when compared to single angle protocols from the far wall alone. ${ }^{14,21,22}$ This may especially be beneficial in settings where sample sizes and effect sizes are small. In addition, from a biological viewpoint it also seems important to measure CIMT at more than one site, as atherosclerosis is asymmetrically distributed across the carotid artery, selectively selecting one angle is likely to ignore the asymmetric nature of the disease. ${ }^{28}$

\section{Reading method}

Ultrasound images in CIMT studies are typically acquired at the study site, stored digitally, and send to a reading laboratory for offline reading. These reading could be performed using different edge detection methods (semi-automatic or manual) and 
a choice should be made between a batch or reading approach.

\section{Semi-automatic or manual readings}

In the early days, all CIMT images were read by readers who manually draw the lines of the lumen and media interfaces on the ultrasound image. However, techniques have advanced and images can now be read using either semi-automated edge detection programs or manual edge detection of the ultrasound interfaces. Semi-automated edge detection is more often applied in settings where only the common carotid artery is examined whereas manual edge detection is usually applied in settings where the carotid bifurcation and the internal carotid artery are additionally measured. ${ }^{10-12,29-32}$ In both approaches a manual selection of the site on the ultrasound image regarding the location of measurements and the area of the arterial segment (i.e. the region of interest) that should be measured is made. The main difference between semi-automated edge detection and manual edge detection, however, is the actual manual drawing of the lines on the interfaces with manual edge detection. With semi-automatic edge detection the reader still may adjust or modify the automatically drawn lines when the reader judges that the lines were incorrectly placed.

A major advantage of semi-automated edge detection programs, besides being less resource intensive and time-consuming, may be the reduction in variability in CIMT readings as a result of reduction in the variability between readers and elimination of change in reading behaviour over time (reader drift). However, a semi-automated edge detection program may be considered a single reader whose behaviour does not change over time. Thus, the potential gain in time and reduction of reader variability with semi-automatic reading depends on how often the automatic outlining of the lumen interfaces is manually modified because of wrong tracing. The more changes, the more the semi-automated program looks like a regular individual reading with all its consequences. Importantly, two recent studies in generally healthy individuals indicated that manual and semiautomated edge detection of lumen and wall interfaces for measurement of far wall common CIMT both resulted in high reproducibility, and largely showed similar relations to cardiovascular risk factors, rates of change, and treatment effects. ${ }^{33,34}$ Hence, choices between semi-automated and manual reading software for CIMT studies likely should be based on logistical and cost considerations rather than differences in expected data quality in populations with a low burden of atherosclerotic disease. However, one may speculate that manual edge detection will perform better than semi-automated edge detection in populations in more advanced stages of atherosclerosis and more irregularities on the ultrasound interfaces.

\section{Batch or random readings}

CIMT images from a single study participant could be read either in batch by a single reader at one point in time or by multiple readers to whom the CIMT images of a particular ultrasound examination are randomly allocated. Batch reading has a logistical drawback that reading cannot start before a particular participant has had the last ultrasound examination and finished the study whereas random reading can be performed continuously during the study. The main disadvantages of continuous random allocation of ultrasound images, however, is that reading behaviour may change over time and that CIMT measured by different readers are additional source of variability. ${ }^{35}$ The batch reading approach, instead, is thought to reduce measurement error in the CIMT measurements as all images of the same participant are read by the same reader and between-reader variability and reader drift (e.g. a change in reading behaviour over time) thus could be substantially reduced. Although both approaches have been used in intervention studies, the most optimal reading approach has never been evaluated (and is not likely to be evaluated) as studies never have used both methods simultaneously. However, an evaluation of the ELSA study of baseline and longitudinal quality control measurements showed a time trend towards lower CIMT values. ${ }^{36}$ Of note, although readers drift results in smaller CIMT values over time, the effect of reader drift on the difference in rate of change (i.e. the outcome of interest) may be small and ignorable if the reader drift is not differential and occurs in all treatment groups to a similar extent.

\section{Sample size}

Sample size calculations for CIMT studies are, as for any study, heavily dependent on the expected magnitude of the rate of change in CIMT, its precision, and the assumed effectiveness of the intervention. The published literature is generally used to obtain these figures. However, determining the most appropriate expected rate of change can be a hard exercise as study populations and interventions vary considerably across studies, with major differences in the reported rates of change in CIMT across studies as a result. ${ }^{37-39}$ Even within groups using a similar study population and intervention, there are major differences in the rates of change in mean common CIMT and mean maximum CIMT. ${ }^{15}$ The rate of change in CIMT is generally larger when mean maximum CIMT is used as primary endpoint as this approach is more affected by carotid plaques than mean common CIMT is. A previous analysis indicated that the rate of change in mean common CIMT was $0.0147 \mathrm{~mm} / \mathrm{y}$, whereas the pooled rate of change in mean maximum CIMT was 0.0176 
$\mathrm{mm} / \mathrm{y}{ }^{40}$ Also, rates of CIMT change are generally larger in individuals with a history of coronary heart disease than in those without. These differences in rates of CIMT change across patient groups represent the differences in natural course of atherosclerosis across carotid segments and between patient groups. These differences are biologically determined and cannot be used as a means to change the required sample size through optimizing the study design. In contrast, differences in the duration of follow-up and the number and timing of ultrasound measurements from for example one to two years could importantly affect the precision of the estimated rates of change and does subsequently also affect the sample size requirements. ${ }^{41,42}$ Study designs with more repeated measurements or a longer duration of follow-up substantially increase the precision and this will decrease the required sample size to detect a given treatment effect with the same level of statistical power. These findings are independent of the expected rate of change in CIMT and the effects of an intervention and thus could be used to optimize the study design relative to costs or other study objectives.

\section{Number of examinations}

Considerable variability in the number of ultrasound examinations and the timing between CIMT examinations can be observed from the published literature. The most basic schemes include single baseline and end-of study examinations alone whereas the most extensive schemes involve duplicate baseline examinations, measurements at regular intervals during the study, and duplicate end-of-study examinations. ${ }^{43}$ The main reason to decrease the number of ultrasound examinations is to reduce the costs and logistics of a study. As CIMT changes linearly over time, the rate of change in CIMT is basically determined by the first and last CIMT measurement. ${ }^{42}$ Thus, the actual effect size is unlikely to be affected by a difference in the number and position of ultrasound examinations within a study. The number of ultrasound examinations, however, has a major effect on the precision of the estimated rate of change, the more examinations the greater the precision. Such increase in precision (and thus in statistical power) may become of critical importance in settings where statistical significance is harder to obtain, that is, in situations where intervention effects or sample sizes are smaller. Besides important improvements in precision, regular ultrasound examinations have a number of additional benefits. First, regular scans also keep the skills of sonographers up-to-date which is likely to improve the quality of the CIMT measurements. ${ }^{44,45}$ Second, when participants drop out, providing at least one post-baseline value, data from these participants can be used in the intention-to-treat analysis. Since dropout rates vary from $10 \%$ to $30 \%$ depending on the type of intervention, this might yield a considerable advantage. Third, duplicate baseline and end-of-study examinations allow for reproducibility studies and will preserve quality control of the data. Finally, intermediate time points allow for assessment of the time course of therapeutic effects which could occur early after initiation of therapy or may not be seen until after prolonged exposure to an effective drug. ${ }^{46}$ These advantages should be carefully considered and weighted against its costs and other study objectives as part of the design of a CIMT study.

\section{Data analyses}

Once the CIMT data has been collected and the images have been read, the next step will be to actually perform the statistical analyses. When analysing CIMT data in intervention studies, one typically describes patterns over time (a rate of change) and characterizes any differences in the rates of change among groups. This can be done by analysing each component of the multivariable data individually or by aggregating all CIMT data in such way that simpler statistical methods such as analysis of variance can be used. Where the first approach introduces concerns about making multiple comparisons, the second approach is not very flexible and typically leads to a loss of information about the natural variability across CIMT measurements. Both approaches should therefore not be recommended. Instead, one should use multivariable approaches which allow for great flexibility in choosing how one models longitudinal data and which can deal with measurements from different carotid sites in one model. ${ }^{47}$ In these linear mixed effects models, the data should be kept hierarchically as study participant, arterial segments within the participant, and measurements over time within participants. With this data format, a linear mixed effects model can be employed which generally includes random effects for the study participant, the CIMT measurement site, and time since randomisation. Fixed effects should be included for at least carotid segment, treatment group, time, and the treatment group by time interaction, the beta coefficient of this interaction term representing the effect of study treatment over time. ${ }^{10-12,32,48} \mathrm{Al}-$ though these linear mixed effects models may be considered more difficult than for example analysis of variances, choices for the statistical model should be balanced between the desire for simplicity in models and the desire to portray data appropriately. As most statistical packages currently include options to use linear mixed effects models, its implementation has become feasible for a broad audience. ${ }^{47,49}$ 


\section{Missing values}

Missing data is a frequently reported problem in CIMT studies and especially the measurements from the carotid bifurcation and internal carotid segments are, as noted above, thought to result in considerable amounts of missing values. ${ }^{16,17,50}$ However, this perception is based on results from early CIMT studies that did not have the rigorous quality control and high quality equipment that is currently available. Indeed, recent studies employing extensive ultrasound protocols have shown marked improvements in data availability with completeness rates for all carotid sites of more than $84 \%$ in high-risk populations and more than $94 \%$ for healthy individuals. ${ }^{19,20}$ Nevertheless, although acquisition of CIMT data becomes more and more complete, missing data cannot be completely excluded. The most common type of missing data in CIMT studies is data that are missing at random, that is, the probability of a measurement being missing is related to other observed variables, such as age, sex, cardiovascular risk factors, or treatment allocation. ${ }^{51,52} \mathrm{In}$ deed, two recent studies indicated that people with a relatively high body mass index had a higher likelihood of missing CIMT data than those with a relatively lower body mass index..$^{19,20}$ If handled inappropriately, these missing CIMT measurements may, even in small amounts, lead to bias in the point estimates and always do affect precision. Multiple imputation of missing values has generally been recommended as approach to deal with missing values as correct use of multiple imputation typically results in unbiased effect estimates and correct standard errors. ${ }^{53,54}$ With multiple imputation, one estimates and imputes the value of the missing CIMT measurement based on the available data resulting in multiple datasets without missing data that are subsequently analysed. ${ }^{55}$ Although multiple imputation is beneficial in many situations, we recently showed in a study using data of the METEOR trial that applying multiple imputation to impute missing CIMT measurements did have no added value in situations where linear mixed effects models are used. ${ }^{56}$ Indeed, in situations where only outcome data (i.e. CIMT measurements) are missing and where the model used to impute the missing values does not provide additional variables that may be used to explain why variables were missing, multiple imputation does not provide any additional benefit over linear mixed effects models. ${ }^{42,56,57}$ However, if there are also missing values in covariables or when the imputation model also includes information on variables that are predictive for missing values that are not included in the linear mixed effects model, multiple imputation still may be a valuable option. Also, multiple imputation is likely to offer benefits in situations where statistical models like analysis of variance are used that are generally less appropriate to model CIMT data.

\section{Biologically implausible values}

Standardization of the ultrasound protocols, training of sonographers and readers, and improved ultrasound equipment have substantially increased the reproducibility of the CIMT measurements over the years..$^{14,21,22,50,58-63}$ However, biologically implausible CIMT values cannot be ruled out and may, if present, seriously affect the effect estimates and precision if handled incorrectly. Biologically implausible CIMT values may arise from natural variation between and within individuals or from routine measurement error. ${ }^{64}$ Natural variation may involve morphological changes such as accelerated increases in CIMT or vessel wall haemorrhages. Measurement error in CIMT measurements may include failure of equipment, deviations from the ultrasound protocol by the sonographer, poor visualization, or errors in the measurement of CIMT ultrasound images by the reader. The principal problem with biologically implausible CIMT values is that there are no established cut-off values or mathematical function to define a biologically implausible value, nor are there appropriate methods to deal with these values. Of course, CIMT values of for example $10 \mathrm{~mm}$ are likely to raise immediate concern, whereas the plausibility of a CIMT of $2.5 \mathrm{~mm}$ is arguable. Thus, deciding on whether or not a value is implausible is rather subjective. In general, there are two options to deal with biologically implausible values. The first is to leave the data as they are and to accept that implausible values are a part of the outcome of the study, or the second is to delete these values from the dataset. ${ }^{65}$ We recently evaluated the effects of deleting implausible values on the estimated rates of change. ${ }^{66}$ We showed that deletion of biologically implausible CIMT values marginally decreased the variability of the estimated rate of change in CIMT without having a large impact on the estimated rate of change. Thus, as defining biologically implausible CIMT values is rather subjective and may be unjustifiable for ethical or scientific reasons, removal of data should be discouraged as long as there is no immediate concern about the plausibility of the data.

\section{Discussion}

In this review, we have provided an up-to-date review of several topics that play an important role in the design and analyses of a CIMT study. The evidence that we showed and considerations that we addressed may serve as a guidance for future intervention studies using CIMT as primary endpoint.

As cardiovascular disease is the leading cause of morbidity and mortality worldwide, the development of targeted new preventive therapies is one of the steps to control the cardiovascular epidemic. Although studies using cardiovascular morbidity 
and mortality as primary endpoint are needed for definite efficacy assessment, these studies are very costly and involve thousands of participants who should be followed for many years. To improve the efficiency of the evaluation of new therapies, alternative endpoints have been sought that provide results within a shorter timeframe, with fewer participants and at lower costs before a large-scale study with cardiovascular morbidity and mortality as endpoint is launched. ${ }^{67} \mathrm{CIMT}$, as a measure of atherosclerosis, is a suitable alternative endpoint for cardiovascular disease events as atherosclerosis is the disease on the pathway between exposure to risk factors and the cause of the majority of cardiovascular events. ${ }^{68,69}$ However, as it is not the change in CIMT itself that leads to prevention of cardiovascular events, the primary prerequisite of the use of CIMT in trials is that CIMT should be measured in a way that best reflects a change in atherosclerosis and cardiovascular risk.

There is no consensus on whether one CIMT protocol is better than another. Some argue that far wall measurements of the common carotid artery alone are the preferred method to study the burden of atherosclerosis using B-mode ultrasound as these measurements have a high reproducibility, are relatively easy and fast to obtain, and have an equally strong relation with the effects of drug therapies as the more extensive protocols have. ${ }^{16,70}$ Nevertheless, there is considerable evidence that the more extensive ultrasound protocols that also include measurements of the near wall and of the carotid bifurcation and the internal carotid artery are the more precise and comprehensive ultrasound protocols. ${ }^{14,15,19-22,43,71}$ Indeed, atherosclerosis develops more rapidly in the carotid bifurcation and the internal carotid artery and the rates of CIMT change are typically larger in these carotid segments than in the common carotid artery. ${ }^{20}$ Importantly, the results from CIMT studies that used an extensive ultrasound protocol were more often in agreement with the findings of the intervention study with cardiovascular morbidity and mortality as primary endpoint than CIMT studies using the common carotid artery alone, suggesting that extensive ultrasound protocols are required to increase the likelihood of success of a study. ${ }^{15}$

There is no concordance on whether a CIMT measurement should include measurement of the thickness of focal carotid plaques if they are present at the site of the measurement. ${ }^{72,73}$ Focal carotid plaques are almost always located in the carotid bifurcation and the internal carotid artery and rarely in the common carotid artery. Carotid plaques are a more advanced stage of atherosclerosis and CIMT measurements including or excluding carotid plaques thus reflect different stages of the atherosclerosis process, i.e. protocols including measurement of carotid plaques having a greater focus on atherosclerotic burden than protocols that only measure CIMT in areas free of plaques.
Nevertheless, a recent consensus statement recommended that CIMT measurements should be taken at sites free of carotid plaque lesions. ${ }^{70}$ The majority of the currently available evidence on CIMT, however, comes from studies in which, when present, regular thickening and plaques were included in the CIMT measurement. ${ }^{10-12,48,74}$ In addition, as CIMT is used as a marker of cardiovascular risk, it seems counterintuitive not to make CIMT measurements in areas in which lesions are present as such approach may be considered as a better representation of the burden of atherosclerosis and cardiovascular risk. ${ }^{75-78}$ This perception is supported by the finding that studies measuring the maximum CIMT at multiple sites are more often in agreement with the findings of the study on clinical events than studies using the mean CIMT value of the common CIMT. ${ }^{15}$

CIMT is a measure of the thickness of the arterial wall that does not tell much about its composition. However, recent studies indicate that the most vulnerable atherosclerotic lesions are not necessarily the largest or thickest lesions, but are the lesions that are rich in lipids pools, have a necrotic core, a thin fibrous cap, and a high macrophage content. ${ }^{79,80}$ As such, techniques that also provide information on the content or composition of the vascular wall may be an important extension to the traditional CIMT measurement. Imaging modalities including carotid magnetic resonance imaging, computed tomography, or coronary intravascular ultrasound have been proposed to serve this purpose ${ }^{67}$ However, these imaging modalities are rather expensive and are not easily applicable to large groups of individuals. Recent advancements in ultrasound techniques, however, including echolucency measurements and vascular elastography are currently being developed and evaluated and are potentially capable in identifying vulnerable lesions in a safe, relatively inexpensive, simple, reliable, and reproducible manner. ${ }^{81-84}$

In conclusion, we recognize that the most optimal approach to design and analyse a CIMT study is very much determined by the research question at hand and the population under study and the present review provides a comprehensive and balanced overview of several aspects that are important to consider during the design and analyses of a CIMT study. Linear mixed effects models are the most appropriate way to analyse CIMT data and handle missing and biologically implausible CIMT values appropriately. Furthermore, we recommend to use extensive CIMT study designs that measure CIMT at regular points during the study at multiple carotid sites as such approach is likely to increase the success rates of CIMT intervention studies designed to evaluate the effects of new interventions on atherosclerotic burden. 


\section{References}

1. Libby P, Ridker PM, Maseri A. Inflammation and atherosclerosis. Circulation 2002;105:1135-1143.

2. Pignoli P, Tremoli E, Poli A, Oreste P, Paoletti R. Intimal plus medial thickness of the arterial wall: a direct measurement with ultrasound imaging. Circulation 1986;74:1399-1406.

3. Lorenz MW, Markus HS, Bots ML, Rosvall M, Sitzer M. Prediction of clinical cardiovascular events with carotid intimamedia thickness: a systematic review and meta-analysis. Circulation 2007;115:459-467.

4. Salonen R, Salonen JT. Determinants of carotid intima-media thickness: a population-based ultrasonography study in eastern Finnish men. J Intern Med 1991;229:225-231.

5. Wang JG, Staessen JA, Li Y, Van Bortel LM, Nawrot T, Fagard $\mathrm{R}$, et al. Carotid intima-media thickness and antihypertensive treatment: a meta-analysis of randomized controlled trials. Stroke 2006;7:1933-1940.

6. Taylor AJ, Kent SM, Flaherty PJ, Coyle LC, Markwood TT, Vernalis MN. ARBITER: Arterial Biology for the Investigation of the Treatment Effects of Reducing Cholesterol: a randomized trial comparing the effects of atorvastatin and pravastatin on carotid intima medial thickness. Circulation 2002;106:20552060.

7. Taylor AJ, Sullenberger LE, Lee HJ, Lee JK, Grace KA. Arterial Biology for the Investigation of the Treatment Effects of Reducing Cholesterol (ARBITER) 2: a double-blind, placebocontrolled study of extended-release niacin on atherosclerosis progression in secondary prevention patients treated with statins. Circulation 2004;110:3512-3517.

8. Crouse JR III, Byington RP, Bond MG, Espeland MA, Craven TE, Sprinkle JW, et al. Pravastatin, Lipids, and Atherosclerosis in the Carotid Arteries (PLAC-II). Am J Cardiol 1995; 5:455459.

9. Crouse JR III, Raichlen JS, Riley WA, Evans GW, Palmer MK, O'Leary DH, et al. Effect of rosuvastatin on progression of carotid intima-media thickness in low-risk individuals with subclinical atherosclerosis: the METEOR Trial. JAMA 2007;297: 1344-1353.

10. Bots ML, Visseren FL, Evans GW, Riley WA, Revkin JH, Tegeler $\mathrm{CH}$, et al. Torcetrapib and carotid intima-media thickness in mixed dyslipidaemia (RADIANCE 2 study): a randomised, double-blind trial. Lancet 2007;370:153-160.

11. Kastelein JJ, van Leuven SI, Burgess L, Evans GW, Kuivenhoven JA, Barter PJ, et al. Effect of torcetrapib on carotid atherosclerosis in familial hypercholesterolemia. N Engl J Med 2007; 356:1620-1630.

12. Kastelein JJ, Akdim F, Stroes ES, Zwinderman AH, Bots ML,
Stalenhoef AF, et al. Simvastatin with or without ezetimibe in familial hypercholesterolemia. N Engl J Med 2008;358:14311443.

13. Zanchetti A, Bond MG, Hennig M, Neiss A, Mancia G, Dal $\mathrm{PC}$, et al. Calcium antagonist lacidipine slows down progression of asymptomatic carotid atherosclerosis: principal results of the European Lacidipine Study on Atherosclerosis (ELSA), a randomized, double-blind, long-term trial. Circulation 2002; 106:2422-2427.

14. Dogan S, Duivenvoorden R, Grobbee DE, Kastelein JJ, Shear $\mathrm{CL}$, Evans GW, et al. Ultrasound protocols to measure carotid intima-media thickness in trials; comparison of reproducibility, rate of progression, and effect of intervention in subjects with familial hypercholesterolemia and subjects with mixed dyslipidemia. Ann Med 2010;42:447-464.

15. Dogan S, Kastelein JJ, Grobbee DE, Bots ML. Mean common or mean maximum carotid intima-media thickness as primary outcome in lipid-modifying intervention studies. J Atheroscler Thromb 2011;18:946-957.

16. Wikstrand J. Methodological considerations of ultrasound measurement of carotid artery intima-media thickness and lumen diameter. Clin Physiol Funct Imaging 2007;27:341-345.

17. Mack WJ, Selzer RH, Hodis HN, Erickson JK, Liu CR, Liu $\mathrm{CH}$, et al. One-year reduction and longitudinal analysis of carotid intima-media thickness associated with colestipol/niacin therapy. Stroke 1993;24:1779-1783.

18. Iglesias del Sol A, Bots ML, Grobbee DE, Hofman A, Witteman JC. Carotid intima-media thickness at different sites: relation to incident myocardial infarction; The Rotterdam study. Eur Heart J 2002; 23:934-940.

19. Dogan S, Duivenvoorden R, Grobbee ED, Kastelein JJ, Shear CL, Evans GW, et al. Completeness of carotid intima media thickness measurements depends on body composition: the RADIANCE 1 and 2 trials. J Atheroscler Thromb 2010;17:526535.

20. Peters SA, den Ruijter HM, Palmer MK, Grobbee DE, Crouse JR III, O'Leary DH, et al. Extensive or restricted ultrasound protocols to measure carotid intima-media thickness: analysis of completeness rates and impact on observed rates of change over time. J Am Soc Echocardiogr 2012;25:91-100.

21. Dogan S, Plantinga Y, Evans GW, Meijer R, Grobbee DE, Bots ML. Ultrasound protocols to measure carotid intima-media thickness: a post-hoc analysis of the OPAL study. Curr Med Res Opin 2009;25:109-122.

22. Dogan S, Plantinga Y, Crouse JR III, Evans GW, Raichlen JS, O'Leary DH, et al. Algorithms to measure carotid intima-media thickness in trials: a comparison of reproducibility, rate of progression and treatment effect. J Hypertens 2011;29:2181- 
2193.

23. Gamble G, Beaumont B, Smith H, Zorn J, Sanders G, Merrilees $\mathrm{M}$, et al. B-mode ultrasound images of the carotid artery wall: correlation of ultrasound with histological measurements. Atherosclerosis 1993;102:163-173.

24. Persson J, Formgren J, Israelsson B, Berglund G. Ultrasounddetermined intima-media thickness and atherosclerosis. Direct and indirect validation. Arterioscler Thromb 1994;14:261-264.

25. Bots ML, de Jong PT, Hofman A, Grobbee DE. Left, right, near or far wall common carotid intima-media thickness measurements: associations with cardiovascular disease and lower extremity arterial atherosclerosis. J Clin Epidemiol 1997;50: 801-807.

26. O’Leary DH, Polak JF, Kronmal RA, Manolio TA, Burke GL, Wolfson SK Jr. Carotid-artery intima and media thickness as a risk factor for myocardial infarction and stroke in older adults. Cardiovascular Health Study Collaborative Research Group. NEngl J Med 1999;340:14-22.

27. Bots ML, Hoes AW, Koudstaal PJ, Hofman A, Grobbee DE. Common carotid intima-media thickness and risk of stroke and myocardial infarction: The Rotterdam study. Circulation 1997;96:1432-1437.

28. Tajik P, Meijer R, Duivenvoorden R, Peters SA, Kastelein JJ, Visseren FJ, et al. Asymmetrical distribution of atherosclerosis in the carotid artery: identical patterns across age, race, and gender. Eur J Prev Cardiol 2012;19:687-697.

29. Hedblad B, Wikstrand J, Janzon L, Wedel H, Berglund G. Lowdose metoprolol $\mathrm{CR} / \mathrm{XL}$ and fluvastatin slow progression of carotid intima-media thickness: main results from the BetaBlocker Cholesterol-Lowering Asymptomatic Plaque Study (BCAPS). Circulation 2001;103:1721-1726.

30. Hodis HN, Mack WJ, LaBree L, Selzer RH, Liu C, Liu C, et al. Reduction in carotid arterial wall thickness using lovastatin and dietary therapy: a randomized controlled clinical trial. Ann Intern Med 1996;124:548-556.

31. Smilde TJ, van WS, Wollersheim H, Trip MD, Kastelein JJ, Stalenhoef AF. Effect of aggressive versus conventional lipid lowering on atherosclerosis progression in familial hypercholesterolaemia (ASAP): a prospective, randomised, double-blind trial. Lancet 2001;357:577-581.

32. Crouse JR III, Grobbee DE, O’Leary DH, Bots ML, Evans GW, Palmer MK, et al. Carotid intima-media thickness in lowrisk individuals with asymptomatic atherosclerosis: baseline data from the METEOR study. Curr Med Res Opin 2007;23: 641-648.

33. Polak JF, Pencina MJ, Herrington D, O’Leary DH. Associations of edge-detected and manual-traced common carotid intima-media thickness measurements with Framingham risk factors: the multi-ethnic study of atherosclerosis. Stroke 2011; 42:1912-1916.

34. Peters SA, den Ruijter HM, Palmer MK, Grobbee DE, Crouse JR III, O'Leary DH, et al. Manual or semi-automated edge detection of the maximal far wall common carotid intima-media thickness: a direct comparison. J Intern Med 2012;271:247-256.

35. Delaney JA, Scherzer R, Polak J, Biggs ML, Kronmal R, Chen $\mathrm{H}$, et al. Effect of inter-reader variability on outcomes in studies using carotid intima media thickness quantified by carotid ultrasonography. Eur J Epidemiol 2010;25:385-392.

36. Tang R, Hennig M, Bond MG, Hollweck R, Mancia G, Zanchetti A. Quality control of B-mode ultrasonic measurement of carotid artery intima-media thickness: the European Lacidipine Study on Atherosclerosis. J Hypertens 2005;23:10471054.

37. Espeland MA, Hoen H, Byington R, Howard G, Riley WA, Furberg CD. Spatial distribution of carotid intimal-medial thickness as measured by B-mode ultrasonography. Stroke 1994;25:1812-1819.

38. Espeland MA, Evans GW, Wagenknecht LE, O'Leary DH, Zaccaro DJ, Crouse JR, et al. Site-specific progression of carotid artery intimal-medial thickness. Atherosclerosis 2003;171: 137-143.

39. Mackinnon AD, Jerrard-Dunne P, Sitzer M, Buehler A, von KS, Markus HS. Rates and determinants of site-specific progression of carotid artery intima-media thickness: the carotid atherosclerosis progression study. Stroke 2004;35:2150-2154.

40. Bots ML, Evans GW, Riley WA, Grobbee DE. Carotid intimamedia thickness measurements in intervention studies: design options, progression rates, and sample size considerations: a point of view. Stroke 2003;34:2985-2994.

41. Peters SA, Palmer MK, den Ruijter HM, Grobbee DE, Crouse I Jr, O'Leary DH, et al. Sample size requirements in trials using repeated measurements and the impact of trial design. Curr Med Res Opin 2012;28:681-688.

42. Fitzmaurice G, Laird N, Ware J. Applied Longitudinal Analysis. Hoboken, NJ: Wiley \& Sons, 2004.

43. Peters SA, Palmer MK, Grobbee DE, Crouse JR III, Evans GW, Raichlen JS, et al. Effect of number of ultrasound examinations on the assessment of carotid intima-media thickness changes over time: the example of the METEOR study. J Hypertens 2011;29:1145-1154.

44. de Groot E, Zwinderman AH, van der Steen AF, Ackerstaff RG, Montauban van Swijndregt AD, Bom N, et al. Variance components analysis of carotid and femoral intima-media thickness measurements. REGRESS study group, Interuniversity Cardiology Institute of the Netherlands, Utrecht, the Netherlands. Regression Growth Evaluation Statin study. Ultra- 
sound Med Biol 1998;24:825-832.

45. de Groot E, Jukema JW, van Boven AJ, Reiber JH, Zwinderman $\mathrm{AH}$, Lie KI, et al. Effect of pravastatin on progression and regression of coronary atherosclerosis and vessel wall changes in carotid and femoral arteries: a report from the Regression Growth Evaluation Statin study. Am J Cardiol 1995;76:40C46C.

46. Bots ML, Palmer MK, Dogan S, Plantinga Y, Raichlen JS, Evans GW, et al. Intensive lipid lowering may reduce progression of carotid atherosclerosis within 12 months of treatment: the METEOR study. J Intern Med 2009;265:698-707.

47. lme4: Linear mixed-effects models using S4 classes [computer program]. 2010.

48. Meuwese MC, de GE, Duivenvoorden R, Trip MD, Ose L, Maritz FJ, et al. ACAT inhibition and progression of carotid atherosclerosis in patients with familial hypercholesterolemia: the CAPTIVATE randomized trial. JAMA 2009;301:11311139.

49. Pinheiro J, Bates D, DebRoy S, Sarkar D; the R Core team. nlme: linear and nonlinear mixed effects models. $R$ package version 3. 1-93. 2009.

50. Furberg CD, Adams HP Jr, Applegate WB, Byington RP, Espeland MA, Hartwell T, et al. Effect of lovastatin on early carotid atherosclerosis and cardiovascular events. Asymptomatic Carotid Artery Progression Study (ACAPS) Research Group. Circulation 1994;90:1679-1687.

51. Little RA, Rubin DB. Statistical Analysis with Missing Data. 2nd ed. New York: J. Wiley \& Sons, 2002.

52. Rubin DB. Multiple imputation for nonresponse in surveys. New York: J. Wiley \& Sons, 1987.

53. DeSouza CM, Legedza AT, Sankoh AJ. An overview of practical approaches for handling missing data in clinical trials. J Biopharm Stat 2009; 19:1055-1073.

54. Donders AR, van der Heijden GJ, Stijnen T, Moons KG. Review: a gentle introduction to imputation of missing values. J Clin Epidemiol 2006;59:1087-1091.

55. Molenberghs G, Kenward MG. Missing data in clinical studies. Chichester: Wiley John \& Sons, 2007.

56. Peters SA, Bots ML, den Ruijter HM, Palmer MK, Grobbee DE, Crouse JR III, et al. Multiple imputation of missing repeated outcome measurements did not add to linear mixed-effects models. J Clin Epidemiol 2012;65:686-695.

57. Espeland MA, Craven TE, Miller ME, D’Agostino RJr. 1996 Remington lecture: modeling multivariate longitudinal data that are incomplete. Ann Epidemiol 1999;9:196-205.

58. Bots ML, Mulder PG, Hofman A, van Es GA, Grobbee DE. Reproducibility of carotid vessel wall thickness measurements. The Rotterdam Study. J Clin Epidemiol 1994;47:921-930.
59. Crouse JR III, Grobbee DE, O’Leary DH, Bots ML, Evans GW, Palmer MK, et al. Measuring effects on intima media thickness: an evaluation of rosuvastatin in subclinical atherosclerosis-the rationale and methodology of the METEOR study Cardiovasc Drugs Ther 2004; 18:231-238.

60. Kastelein JJ, van Leuven SI, Evans GW, Riley WA, Revkin JH, Shear CL, et al. Designs of RADIANCE 1 and 2: carotid ultrasound studies comparing the effects of torcetrapib/atorvastatin with atorvastatin alone on atherosclerosis. Curr Med Res Opin 2007;23:885-894.

61. Espeland MA, Craven TE, Riley WA, Corson J, Romont A, Furberg CD. Reliability of longitudinal ultrasonographic measurements of carotid intimal-medial thicknesses. Asymptomatic Carotid Artery Progression Study Research Group. Stroke 1996;27:480-485.

62. Salonen R, Haapanen A, Salonen JT. Measurement of intimamedia thickness of common carotid arteries with high-resolution B-mode ultrasonography: inter- and intra-observer variability. Ultrasound Med Biol 1991;17:225-230.

63. Riley WA, Barnes RW, Applegate WB, Dempsey R, Hartwell T, Davis VG, et al. Reproducibility of noninvasive ultrasonic measurement of carotid atherosclerosis. The Asymptomatic Carotid Artery Plaque study. Stroke 1992;23:1062-1068.

64. Meijer R, Stork S, Evans GW, Grobbee DE, Bots ML. Striking increases in carotid artery wall thickness in healthy subjects. Cerebrovasc Dis 2010;30:448-455.

65. Ludbrook J. Outlying observations and missing values: how should they be handled? Clin Exp Pharmacol Physiol 2008;35: 670-678.

66. Peters SA, den Ruijter HM, Palmer MK, Grobbee DE, Crouse JR III, O'Leary DH, et al. Biologically implausible carotid intima-media thickness measurement values: effects on rate of change over time. Curr Med Res Opin 2012;28:891-899.

67. Duivenvoorden R, de GE, Stroes ES, Kastelein JJ. Surrogate markers in clinical trials-challenges and opportunities. Atherosclerosis 2009;206:8-16.

68. Peters SA, den Ruijter HM, Bots ML. Attenuation of rate of change in carotid intima-media thickness by lipid-modifying drugs: impact on clinical outcomes. Am J Cardiovasc Drugs 2011;11:253-263.

69. Peters SA, Grobbee DE, Bots ML Carotid intima-media thickness: a suitable alternative for cardiovascular risk as outcome? Eur J Cardiovasc Prev Rehabil 2011;18:167-174.

70. Stein JH, Korcarz CE, Hurst RT, Lonn E, Kendall CB, Mohler $\mathrm{ER}$, et al. Use of carotid ultrasound to identify subclinical vascular disease and evaluate cardiovascular disease risk: a consensus statement from the American Society of Echocardiography Carotid Intima-Media Thickness Task Force. Endorsed 
by the Society for Vascular Medicine. J Am Soc Echocardiogr 2008;21:93-111.

71. Peters SA, den Ruijter HM, Bots ML. Ultrasound protocols to measure carotid intima-media thickness: one size does not fit all. J Am Soc Echocardiogr 2012;25:1135-1137.

72. Touboul PJ, Hennerici MG, Meairs S, Adams H, Amarenco P, Bornstein N, et al. Mannheim carotid intima-media thickness consensus (2004-2006). An update on behalf of the Advisory Board of the 3rd and 4th Watching the Risk Symposium, 13th and 15th European Stroke Conferences, Mannheim, Germany, 2004, and Brussels, Belgium, 2006. Cerebrovasc Dis 2007;23: 75-80.

73. Finn AV, Kolodgie FD, Virmani R. Correlation between carotid intimal/medial thickness and atherosclerosis: a point of view from pathology. Arterioscler Thromb Vasc Biol 2010;30:177-181.

74. O'Leary DH, Bots ML. Imaging of atherosclerosis: carotid intima-media thickness. Eur Heart J 2010;31:1682-1689.

75. Brook RD, Bard RL, Patel S, Rubenfire M, Clarke NS, Kazerooni EA, et al. A negative carotid plaque area test is superior to other noninvasive atherosclerosis studies for reducing the likelihood of having underlying significant coronary artery disease. Arterioscler Thromb Vasc Biol 2006;26:656-662.

76. Ebrahim S, Papacosta O, Whincup P, Wannamethee G, Walker $\mathrm{M}$, Nicolaides AN, et al. Carotid plaque, intima media thickness, cardiovascular risk factors, and prevalent cardiovascular disease in men and women: the British Regional Heart study. Stroke 1999;30:841-850.

77. Johnsen SH, Mathiesen EB, Joakimsen O, Stensland E, Wilsgaard T, Lochen ML, et al. Carotid atherosclerosis is a stronger predictor of myocardial infarction in women than in men: a 6-year follow-up study of 6226 persons: the Tromso study. Stroke 2007;38:2873-2880.

78. Rundek T, Arif H, Boden-Albala B, Elkind MS, Paik MC, Sacco RL. Carotid plaque, a subclinical precursor of vascular events: the Northern Manhattan Study. Neurology 2008;70:1200-1207.

79. Naghavi M, Libby P, Falk E, Casscells SW, Litovsky S, Rumberger J, et al. From vulnerable plaque to vulnerable patient: a call for new definitions and risk assessment strategies: part I. Circulation 2003; 108:1664-1672.

80. Naghavi M, Falk E, Hecht HS, Jamieson MJ, Kaul S, Berman $D$, et al. From vulnerable plaque to vulnerable patient-Part III: Executive summary of the Screening for Heart Attack Prevention and Education (SHAPE) Task Force report. Am J Cardiol 2006;98:2H-15H.

81. de Korte CL, Hansen HH, van der Steen AF. Vascular ultrasound for atherosclerosis imaging. Interface Focus 2011;1:565575 .

82. Lind L, Peters SA, den Ruijter HM, Palmer MK, Grobbee DE, Crouse JR III, et al. Effect of rosuvastatin on the echolucency of the common carotid intima-media in low-risk individuals: the METEOR trial. J Am Soc Echocardiogr 2012;25: 1120-1127.

83. Peters SA, Lind L, Palmer MK, Grobbee DE, Crouse JR III, O'Leary DH, et al. Increased age, high body mass index and low HDL-C levels are related to an echolucent carotid intimamedia: the METEOR study. J Intern Med 2012;272:257-266.

84. Gronholdt ML, Nordestgaard BG, Schroeder TV, Vorstrup S, Sillesen $\mathrm{H}$. Ultrasonic echolucent carotid plaques predict future strokes. Circulation 2001;104:68-73. 